\title{
The ultraluminous X-ray source NGC 1313 X-2
}

\section{Its optical counterpart and environment}

\author{
F. Grisé ${ }^{1}$, M. W. Pakull ${ }^{1}$, R. Soria ${ }^{2}$, C. Motch $^{1}$, I. A. Smith ${ }^{3}$, S. D. Ryder ${ }^{4}$, and M. Böttcher ${ }^{5}$ \\ 1 Observatoire Astronomique de Strasbourg, 11 rue de l'Université, 67000 Strasbourg, France \\ e-mail: grise@astro.u-strasbg.fr \\ 2 Mullard Space Science Laboratory (UCL), Holmbury St Mary, Dorking, Surrey RH5 6NT, UK \\ 3 Department of Physics and Astronomy, Rice University, 6100 South Main, MS108, Houston, TX 77251-1892, USA \\ 4 AngloAustralian Observatory, PO Box 296, Epping, NSW 1710, Australia \\ 5 Astrophysical Institute, Department of Physics and Astronomy, Ohio University, Clippinger Hall 251B, Athens, OH 45701-2979, \\ USA
}

Received 11 February 2008 / Accepted 18 May 2008

\section{ABSTRACT}

\begin{abstract}
NGC $1313 \mathrm{X}-2$ is one of the brightest ultraluminous X-ray sources in the sky, at both X-ray and optical wavelengths; therefore, quite a few studies of available ESO VLT and HST data have appeared in the literature. Here, we present our analysis of VLT/FORS1 and HST/ACS photometric data, confirming the identification of the $B \sim 23$ mag blue optical counterpart. We show that the system is part of a poor cluster with an age of $20 \mathrm{Myr}$, leading to an upper mass limit of some $12 M_{\odot}$ for the mass donor. We attribute the different results with respect to earlier studies to the use of isochrones in the F435W and F555W HST/ACS photometric system that appear to be incompatible with the corresponding Johnson $B$ and $V$ isochrones. The counterpart exhibits significant photometric variability of about 0.2 mag amplitude, both between the two HST observations and during the one month of monitoring with the VLT. This includes variability within one night and suggests that the light is dominated by the accretion disk in the system and not by the mass donor.
\end{abstract}

Key words. galaxies: individual: NGC 1313 - accretion, accretion disks - black hole physics - galaxies: star clusters X-rays: binaries - X-rays: individuals: NGC $1313 \mathrm{X}-2$

\section{Introduction}

\subsection{Context}

Ultraluminous X-ray sources (ULXs) are extragalactic X-ray sources that are not at the nucleus of their galaxy, emitting well above the Eddington limit of a $10 M_{\odot}$ black hole $\left(L_{X} \sim\right.$ $10^{39} \mathrm{erg} \mathrm{s}^{-1}$ ) if we assume that they emit isotropically. An important question is whether they contain intermediate mass black holes (Colbert \& Mushotzky 1999; Makishima et al. 2000), whether they are beamed (King et al. 2001), or if they are rather normal X-ray binaries with super-Eddington emission (Begelman 2002).

The first clues about their compact nature came from X-ray studies showing that ULXs are variable on timescales of years down to minutes. Well-studied examples are Holmberg IX X-1 (La Parola et al. 2001), ULXs in the Antennae (Fabbiano et al. 2003) or M74 X-1 (Krauss et al. 2005). Therefore, ULXs are accreting systems displaying spectra that were first described by a soft multicolor disk blackbody plus power-law continuum (Miller et al. 2003). The soft component was interpreted as a cool accretion disk $(k T \sim 150 \mathrm{eV})$. Using standard (Shakura \& Syunyaev 1973) multicolor disk models $\left(M \sim L^{1 / 2} T_{\text {in }}^{-2}\right)$, this would imply black hole (BH) masses $\sim 10^{3}-10^{4} M_{\odot}$ assuming that the disk extends to the innermost stable circular orbit (as is thought to be the case in Galactic black-hole X-ray binaries in the disk-dominated high/soft state). However, it has been suggested (Roberts 2007; Soria 2007) that the assumptions might well be misleading: the observed energy distribution can be interpreted with alternative spectral models based on a cool outer disk and a Compton-scattering-dominated inner region. In this scenario, much lower masses $\left(\lesssim 100 M_{\odot}\right)$ are required for most ULXs, even in the absence of strong beaming.

Further clues to the ULX nature come from X-ray timevariability studies. The detection of low-frequency quasiperiodic oscillations (LF-QPOs) in a few ULXs exclude strong X-ray beaming (Strohmayer \& Mushotzky 2003; Dewangan et al. 2006; Mucciarelli et al. 2006; Strohmayer et al. 2007). By analogy with Galactic BHs and with some AGNs (McHardy et al. 2006), the characteristic frequencies of the LF-QPOs and of the breaks observed in the power-density-spectra of a few ULXs suggest masses in the intermediate mass black hole (IMBH) range (Strohmayer et al. 2007; Shaposhnikov \& Titarchuk 2007), if the varying component is emitted at or near the innermost stable circular orbit. However, as for the spectral interpretation, lower BH masses would be implied if the oscillations come from a larger region, perhaps associated with the thermal/non-thermal transition of the accretion flow. On longer timescales (weeks to years), spectral state transitions have been observed in a few luminous sources (e.g. La Parola et al. 2001; Soria \& Motch 2004; Soria et al. 2007), but it is not yet clear how they compare with the "canonical" state transitions in Galactic BHs (e.g. Remillard et al. 2003).

Optical studies of ULXs have revealed point-like counterparts with blue colors (Goad et al. 2002; Liu et al. 2002; Kaaret et al. 2004; Liu et al. 2004; Soria et al. 2005; Kuntz et al. 2005; 
Mucciarelli et al. 2005; Grisé et al. 2006; Liu et al. 2007; Rosado et al. 2008), consistent with early-type donors (and more specifically, with 10-20 $M_{\odot}$ blue supergiants). However, the optical emission may have a strong, perhaps dominant contribution from the accretion disk (which should also have blue colors). This makes it more difficult to identify the spectral type and mass of the donor star from the luminosity and colors of the optical counterpart.

In many cases, optical counterparts are embedded in highlysupersonically expanding ionized nebulæ (with possible contributions due to photoionization) and possibly related to the presence of jets (Pakull \& Mirioni 2002, 2003; Kaaret et al. 2004; Abolmasov et al. 2007a,b; Pakull \& Grisé 2008). The study of nearby ULXs undergoing little interstellar extinction revealed that stellar counterparts are usually part of small young star clusters or OB associations (Zezas et al. 2002; Goad et al. 2002; Grisé et al. 2006; Liu et al. 2007; Abolmasov et al. 2007b). But they are sometimes found slightly separated from these stellar associations (Zezas et al. 2002), as if they received a kick during a supernova event (which in this case could strengthen the stellar mass nature of the black hole). Radio counterparts are found in only few cases (Miller et al. 2005; Soria et al. 2006; Lang et al. 2007). They are spatially resolved, and have a spectrum consistent with optically-thin (steep) synchrotron emission. Thus, they are likely to be jet-powered radio lobes, not core emission from the collimated inner jet as observed in microquasars.

ULXs are mostly found in star-forming spirals or irregular galaxies (Irwin et al. 2004; Swartz et al. 2004). Because of their extragalactic nature, these sources are optically faint. Telescopes with large collecting area such as the VLT and/or high spatial resolution such as HST are therefore required for the study of their optical counterparts and immediate environments.

\subsection{NGC $1313 X-2$ ULX}

Here, we study ULX X-2 in the barred spiral galaxy NGC 1313 , which is located at a distance of about $4 \mathrm{Mpc}$ (3.7 Mpc according to Tully 1988, 4.13 Mpc according to Méndez et al. 2002). NGC 1313 has a mass of $M=10^{10.25} M_{\odot}$ (Karachentsev et al. 2004); this is roughly in the mass range where galaxies appear to be most efficient at producing ULXs (Swartz et al. 2008). X-2 is quite distant from the galactic nucleus, $\approx 6^{\prime}$ (corresponding to $7 \mathrm{kpc}$ ) to the south. At this position, there are no obvious signs of recent, extensive star formation. The galactic interstellar (IS) extinction is low $(E(B-V) \sim 0.1$, Schlegel et al. 1998): this makes X-2 one of the best targets for an optical spectroscopic and photometric study of a ULX and its host environment.

\subsubsection{X-ray properties}

The source has been extensively studied in X-rays (see Feng \& Kaaret 2006, for a study of 12 archival XMM-Newton observations; Mizuno et al. 2007, for a recent Suzaku study), and has an average isotropic luminosity of $\approx 6 \times 10^{39} \mathrm{erg} \mathrm{s}^{-1}$ in the $0.3-10 \mathrm{keV}$ band. It was seen to vary in intensity by about $50 \%$ in a few hours (Mizuno et al. 2007) and exhibits also significant variability on time scales of months and years. The X-ray spectrum of X-2 can be fitted by at least 2 phenomenological models corresponding to 3 alternative physical mechanisms:

a) a hot thermal component $\left(k T_{\text {in }} \approx 1.2-1.3 \mathrm{keV}\right)$ dominating above $2 \mathrm{keV}$, plus a soft down-scattered component. The hot thermal component was physically interpreted as a "slim disk" around a stellar-mass black hole, at super-Eddington accretion rates (Mizuno et al. 2007);

b) a cool thermal component $\left(k T_{\text {in }} \approx 0.15 \mathrm{keV}\right)$ plus a powerlaw-like component dominating above $2 \mathrm{keV}$. Physically, this can be interpreted in two ways:

i) as an optically-thick accretion disk extending to the innermost stable circular orbit, plus an optically-thin corona, around an intermediate-mass black hole $\left(M \sim 1000 M_{\odot}\right)$, with accretion rates below 0.1 times Eddington (Miller et al. 2003);

ii) as an optically-thick disk, directly visible only far away from the innermost stable orbit, and replaced (or covered) at smaller radii by a hotter region $(k T \sim$ a few $\mathrm{keV})$, optically-thick to electron scattering. This scenario may allow for less massive black holes $\left(\lessgtr 100 M_{\odot}\right)$ at accretion rates $\sim$ a few times Eddington (Roberts 2007; Soria 2007).

These three physical models predict different characteristic sizes, colors and luminosities for the accretion disk. Optical studies can provide new constraints, if we can disentangle the optical contributions from the irradiated donor star and the accretion disk.

\subsubsection{Optical properties}

At optical wavelengths, studies by Pakull \& Mirioni (2002); Pakull et al. (2006); Ramsey et al. (2006) revealed the presence of a huge ionized nebula (extension $18^{\prime \prime} \times 26^{\prime \prime}$, corresponding to $350 \times 500 \mathrm{pc}$ at a distance of $4.0 \mathrm{Mpc}$ ) at the position of X-2. The high expansion velocity of $\sim 100 \mathrm{~km} \mathrm{~s}^{-1}$ (Ramsey et al. 2006) underlines the suggestion that we are seeing emission from radiative shocks. This is supported by the presence of enhanced [OI] and [SII] forbidden lines (e.g. Pakull \& Mirioni 2002; Abolmasov et al. 2007a,b). However, contributions due to photoionization by the X-ray source cannot presently be excluded. The search for an optical counterpart of the ULX has motivated to carry out deep imaging of the field around it, using the European Southern Observatory Very Large Telescope (ESO/VLT) and the Hubble Space Telescope Advanced Camera for Surveys (HST/ACS).

Multi-band photometry of the counterpart and of the surrounding stellar population can constrain the nature of the donor star, but results reported in the literature are quite discordant (see Mucciarelli et al. 2005, 2007; Ramsey et al. 2006; Liu et al. 2007). In particular, the age of the small cluster or association of relatively young stars around the ULX appears not to be well constrained. Determining this value is of great interest, not at least because it tells us whether or not the ULX donor is a massive early-type star, and hence helps to constrain the physics and duration of the high mass-transfer phase.

In this paper, we analyze the full set of our photometric VLT observations (some results were previously reported in Pakull et al. 2006) together with archival HST/ACS data. We will show that the ULX counterpart is part of a 20-Myr-old star cluster, which is not expected to contain uncollapsed stars more massive than $\approx 12 M_{\odot}$. Combining ground-based and HST data, we will present a detailed photometric light curve of the ULX counterpart, which shows large variability on timescales of hours and days. This suggests that the optical emission is dominated by an accretion disk with possible contributions by the X-ray-heated secondary. 
Table 1. The VLT FORS1 observations for NGC 1313 X-2.

\begin{tabular}{lcccc}
\hline \hline Filter & Exposure time $(\mathrm{s})$ & Number of exposures & Central wavelength of filter $(\AA)$ & $F W H M$ of filter $(\AA)$ \\
\hline$B$ & 420 & 24 & 4290 & 880 \\
& 840 & 4 & & \\
$V$ & 600 & 2 & 5540 & 1115 \\
$R$ & 500 & 4 & 6570 & 1500 \\
$\mathrm{H}_{\alpha}$ & 1500 & 2 & 6563 & 61 \\
{$[\mathrm{OI}]$} & 1300 & 2 & 6295 & 69 \\
{$[\mathrm{OIII}]$} & 1500 & 2 & 5001 & 55 \\
\hline
\end{tabular}

\section{Observations}

\subsection{VLT observations}

The first set of data comes from an observing programme (ID 072.D0614; PI: M. Pakull) carried out with the VLT/FORS1 instrument. Photometric monitoring was done over 9 nights and a spectroscopic study over 4 nights, between 2003 December 20 and 2004 January 15 . The field around X-2 was observed in broadband filters (standard $B, V, R$ ) and in some narrow-band filters $\left(\mathrm{H}_{\alpha},[\mathrm{OIII}] \lambda 5007\right.$ and $\left.[\mathrm{OI}] \lambda 6300\right)$ (see Tables 1 and 2 for details). We also monitored the source in the $B$ band with a total of 16 observations, each with an exposure time of $840 \mathrm{~s}$. Usually, we obtained one observation per night, except for 2003 December 24, when the field was observed 7 times. Each observation consisted of two dithered sub-exposures taken directly one after the other, and averaged to increase the signal-tonoise ratio. We also obtained two observations in $R$ and one in $V$ to study the color of the source and of its stellar environment. This paper mainly concentrates on photometric results; we leave the discussion of the spectroscopic results to a subsequent paper.

\subsection{HST observations}

The second dataset was obtained from the HST/ACS archive (programme GO-9796; PI: J. Miller). Observations were carried out on 2003 November 22, with the Wide Field Camera (WFC) in the $F 435 W, F 555 W$ and $F 814 W$ filters, and with the High Resolution Camera (HRC) in the F330W filter. An additional observation was done on 2004 February 22 with the WFC in the $F 555 \mathrm{~W}$ filter. More detailed information about those exposures is summarized in Table 3 . We re-analyzed the data using the latest calibration files at the time of the analysis (STSDAS v3.5) and applied the standard correction for the spatial distortion (Multidrizzle 2.7.2, Koekemoer et al. 2002).

\section{Data analysis}

\subsection{VLT data}

The near environment of X-2 is crowded, so in order to obtain the best possible photometric measurements, we used point-spread-function (PSF) fitting routines in DAOPHOT II (Stetson 1992), a sub-package of the MIDAS photometry software. We calibrated our absolute photometry using the exposures from 2003 December 24 for the $B$ and $R$ bands, and 2003 December 25 for the $V$ band; both nights were photometric. Service mode observations also included standard star observations of the Landolt fields PG0231 and SA101; our transformations from the instrumental to the $B V R$ system are fully consistent with the zeropoints and color terms provided by ESO.
Table 2. Log of our VLT FORS1 broad-band observations.

\begin{tabular}{lccc}
\hline \hline Filter & Observation date & MJD at mid-exposure & Seeing $\left(^{\prime \prime}\right)$ \\
\hline$B$ & 2003 Dec. 21 & 52994.12864 & 0.60 \\
& 2003 Dec. 22 & 52995.04826 & 0.56 \\
& 2003 Dec. 23 & 52996.08222 & 0.66 \\
& 2003 Dec. 24 & 52997.03231 & 0.58 \\
& & 52997.08794 & 0.68 \\
& & 52997.09836 & 0.71 \\
& & 52997.12389 & 0.67 \\
& & 52997.20328 & 0.69 \\
& & 52997.21365 & 0.76 \\
& & 52997.24293 & 0.50 \\
& 2003 Dec. 25 & 52998.17256 & 0.46 \\
& 2003 Dec. 27 & 53000.22022 & 0.70 \\
& 2003 Dec. 28 & 53001.17035 & 0.69 \\
$V$ & 2003 Dec. 29 & 53002.21072 & 0.58 \\
$R$ & 2003 Dec. 30 & 53003.04551 & 0.65 \\
& 2004 Jan. 15 & 53019.04868 & 0.57 \\
& 2003 Dec. 25 & 52998.07801 & 0.55 \\
& 2003 Dec. 24 & 52997.11028 & 0.75 \\
& & 52997.22568 & 0.80 \\
\hline
\end{tabular}

\subsection{HST data}

Our field of interest (Fig. 1) also appears moderately crowded in the HST/ACS drizzled images, so we used again DAOPHOT II for our photometric analysis. In order to obtain a realistic estimate of the photometric errors, we multiplied the pixel values by the exposure time, which gave us the number of detected $\mathrm{e}^{-}$per pixel; we then added the background counts subtracted by the HST pipeline. We selected a sample of bright, isolated stars in the field to model the PSF; we allowed the PSF to vary quadratically as a function of spatial position in the frame. We used a radius of 3 pixels for PSF fitting to the other stars. We carried out aperture photometry of isolated stars with SExtractor (Bertin \& Arnouts 1996) in order to calculate the aperture corrections between PSF-derived brightnesses and those from a 0.5 aperture radius (Table 5). Finally we applied the values given in Sirianni et al. (2005) to correct between 0.'5 aperture and infinite aperture photometry.

From the ACS instrumental magnitudes, we calculated the standard magnitudes in both the VEGAMAG and the JohnsonCousins systems. We are aware of the limitations of such transformations, especially for stars with peculiar spectral features. But we chose to do so in order to facilitate a comparison between the ground-based and HST photometry. Although, in principle, it should not be necessary to transform between these sets of magnitudes (Sirianni et al. 2005), it turns out (as we discuss in Sect. 4.5) that the interpretation of the data in terms of published isochrones differs substantially between the $(F 435 \mathrm{~W}$, $F 555 W)$ system on the one hand and the $(B, V)$ system on the other. In order to check the transformations given by Sirianni et al. (2005) between these two photometric systems, we used 
Table 3. The HST/ACS observations for NGC 1313 X-2.

\begin{tabular}{lcccccc}
\hline \hline ID & Instruments & Filter & Date & MJD at mid-exposure & Exposure time $(\mathrm{s})$ & $F W H M$ of filter $(\AA)$ \\
\hline j8ola2010 & HRC & $F 330 W$ & 2003 Nov. 22 & 52965.44294 & 2760 & 173.82 \\
j8ol02040 & WFC & $F 435 W$ & 2003 Nov. 22 & 52965.37542 & 2520 & 293.47 \\
j8ol02030 & WFC & $F 555 W$ & 2003 Nov. 22 & 52965.31531 & 1160 & 360.02 \\
j8ol02010 & WFC & $F 814 W$ & 2003 Nov. 22 & 52965.30656 & 1160 & 654.64 \\
j8ol06010 & WFC & $F 555 W$ & 2004 Feb. 22 & 53057.22947 & 2240 & 360.02 \\
\hline
\end{tabular}
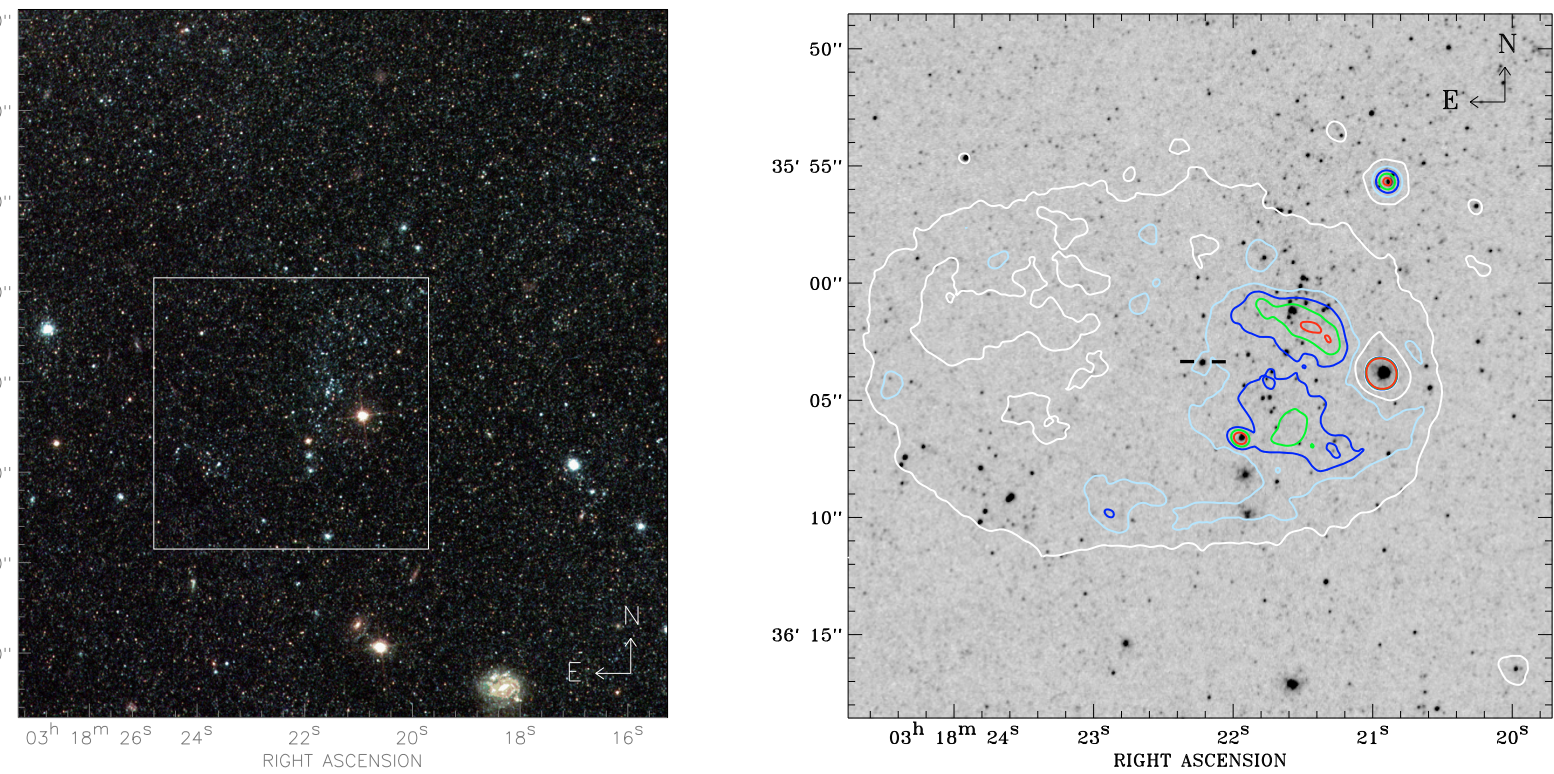

Fig. 1. Left panel: true color image (blue $=F 435 W$; green $=F 555 W$; red $=F 814 W$ ) of the region around X-2, from our HST/ACS observations. $1^{\prime \prime}$ represents $19.4 \mathrm{pc}$ at the distance of NGC 1313. The stellar environment is mainly composed of red stars from an old population. Two blue-star associations can be seen: the main one to the west of the ULX counterpart and the other (smaller) one to the south-east. Right panel: zoomed-in view of the immediate vicinity of the counterpart, in the $F 435 \mathrm{~W}$ filter. The ULX counterpart is the bright point-like source at the center of the image. Contours of the $\mathrm{H}_{\alpha}$ emission (at 10, 30, 50, 70 and 90\% flux level above the background) are overplotted, from our VLT observations. Both young stellar associations are located or projected inside the $\mathrm{H} \alpha$ nebula.

our data to derive our own calibration. We selected bright stars in the VLT image that are not saturated or resolved into multiple stars by ACS. We found that our own set of transformations are consistent with those of Sirianni et al. (2005), which we use for consistency with other studies. We interpreted our color-magnitude diagrams in the two photometric systems by comparing them with evolutionary tracks and isochrones from the Padua group (Bertelli et al. 1994; Girardi et al. 2000; Salasnich et al. 2000; Girardi et al. 2002) and the Geneva group (Lejeune \& Schaerer 2001).

We estimated the completeness limit in all frames by adding 1000 artificial stars in each 0.2 mag bin between 24.8 mag (where the completeness is 1 in all filters) and $28.6 \mathrm{mag}$ (where the completeness is 0 for all filters). For each bin we took the mean of the number of stars recovered in three runs as the completeness fraction.

\section{Results and discussion}

\subsection{Distance and metallicity of NGC 1313}

The HST spatial resolution is required for any stellar population study in NGC 1313 (see Méndez et al. 2002; Pellerin et al. 2007; Rizzi et al. 2007, for recent work). The central region of the galaxy is dominated by young stellar populations; by contrast the field around X-2 contains few young stars and appears to be dominated by an older population. This is clearly shown in our
$(V-I, I)$ color-magnitude diagram (Fig. 2) of the $\approx 7200$ stars identified in the ULX neighbourhood (the region around X-2 shown in Fig. 1).

For old stellar populations, the location of the red giant branch (RGB) in color-magnitude diagrams (in particular, $(V-$ $I, I)$ ) is a good indicator of distance and metallicity. In our case, the RGB is very prominent. We estimated the position of its tip (TRGB) by determining the point at which the first derivative of the $I$-band luminosity function has a maximum. We find that it occurs at $I_{0 \text { (TRGB) }} \approx 24.0 \pm 0.1 \mathrm{mag}$ (after correcting for extinction). The distance can be expressed by:

$(m-M)_{0}=I_{0(\mathrm{TRGB})}-M_{I_{\mathrm{TRGB}}}$

where $(m-M)_{0}$ is the distance modulus and $M_{I_{\mathrm{TRGB}}}$ is the absolute magnitude of the TRGB. Taking $M_{I_{\mathrm{TRGB}}}=-4.05 \pm 0.02$ (Rizzi et al. 2007), we find that the distance modulus to NGC 1313 is $(m-M)_{0} \approx 28.05 \pm 0.11$, corresponding to a distance of $4.07 \pm$ $0.22 \mathrm{Mpc}$. This is in agreement with the results of Méndez et al. (2002) and Rizzi et al. (2007) who studied the north-western region of the galaxy (distance modulus of $28.08 \pm 0.06$ mag and $28.15 \pm 0.03 \mathrm{mag}$, respectively).

The intrinsic color of the RGB depends on the metal content of its stars (Da Costa \& Armandroff 1990). A useful empirical relation between color at $M_{I}=-3.5 \mathrm{mag}$ (half a magnitude below the tip) and metal abundance is

$[\mathrm{Fe} / \mathrm{H}]=-12.64+12.61(V-I)_{0,-3.5}-3.33\left[(V-I)_{0,-3.5}\right]^{2}$. 


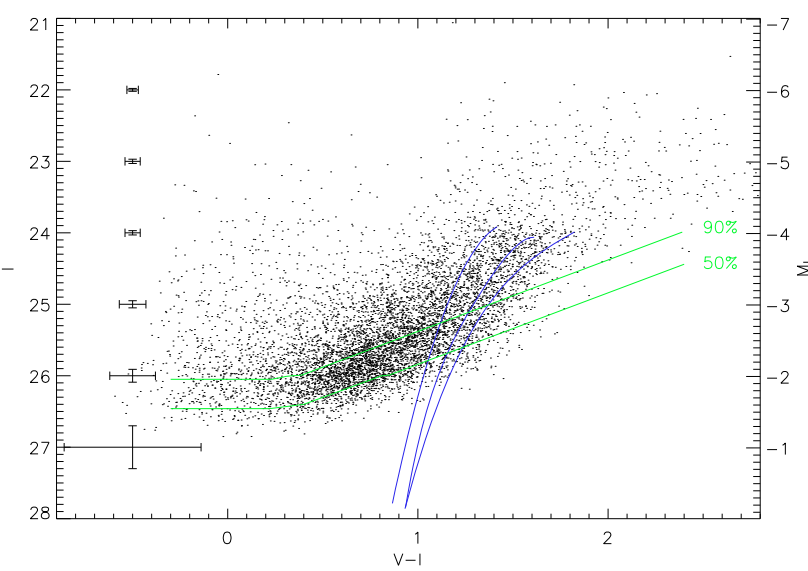

Fig. 2. $(V-I, I)$ color-magnitude diagram of all the stars located in the ACS field (see Fig. 1, left panel). The estimated 50\% and 90\% completeness limits are overplotted, as are the RGB loci of three Galactic globular clusters of various metallicities (from left to right: M 15, NGC 6752 and NGC 1851; data from Da Costa \& Armandroff 1990).

Table 4. Galactic globular clusters used for comparison with NGC 1313.

\begin{tabular}{lcc}
\hline \hline Cluster & {$[\mathrm{Fe} / \mathrm{H}]$} & $M_{I, \mathrm{TRGB}}$ \\
\hline NGC 7078 (M 15) & -2.17 & -4.095 \\
NGC 6752 & -1.54 & -3.948 \\
NGC 1851 & -1.29 & -4.039 \\
\hline
\end{tabular}

(Lee et al. 1993). To highlight the metallicity dependence, we overplotted the RGB loci (scaled to the distance of NGC 1313) of galactic globular clusters (Da Costa \& Armandroff 1990) with different metal abundances (Fig. 2 and Table 4).

We took horizontal cuts across the RGB at two magnitudes: $I=24.5 \pm 0.1 \mathrm{mag}, I=25.0 \pm 0.1 \mathrm{mag}$. We determined the number distributions at those magnitudes, and corrected them for the completeness fraction. We can clearly identify the number peak in both histograms (Fig. 3) and their decline towards redder values of $V-I$. This suggests that we are seeing the central locus of the RGB in NGC 1313. We estimate $(V-I)_{0,-3.5}=$ $(1.30 \pm 0.08)$ mag (left panel of Fig. 3).

Using the relation in Lee et al. (1993), we infer $[\mathrm{Fe} / \mathrm{H}] \approx$ $-1.9 \pm 0.3$, which is what we qualitatively expect from an old population at the outskirts or in the halo of a disk galaxy. For example, a relation between metal abundance of the halo population and absolute $V$ magnitude of the host galaxy was highlighted by Mouhcine et al. (2005) (in particular, their Fig. 3). For NGC 1313, which has $M_{V}=-18.72 \mathrm{mag}$ (de Vaucouleurs et al. 1991), the expected metal abundance of its halo is $[\mathrm{Fe} / \mathrm{H}] \approx$ $-2--1.5$, in agreement with the abundance we found for the old population in the ULX field.

At fainter magnitudes, $M_{V} \gtrsim-2.5 \mathrm{mag}$, the RGB is very spread out and there is a substantial contribution from stars with $0.5 \lesssim(V-I)_{0} \lesssim 1.0$ (Fig. 3, right panel). They are consistent with a population of younger stars, with ages around 1-2 Gyr. Star formation in the field surrounding the ULX may have had various episodes until as recently as $1 \mathrm{Gyr}$, before the last, localized episode, responsible for the formation of the few young stars around the ULX (Fig. 1 and Sect. 4.3.1).

\subsection{Identification of the optical counterpart}

In earlier studies of this ULX (Mucciarelli et al. 2005), two possible optical counterparts, named $\mathrm{C} 1$ and $\mathrm{C} 2$, were suggested. A more accurate astrometric calibration of the Chandra and HST images pointed in favour of C1 (Ramsey et al. 2006; Liu et al. 2007). The two objects are separated by 0.9 , i.e. 16 ACS pixels. More importantly and contrary to the claims of Mucciarelli et al. (2005), we found (Pakull et al. 2006) that they can also be clearly resolved in our VLT spectra with a projected distance of 4 pixels corresponding to 0 .' 5 . The characteristic high-excitation emission line spectrum displaying a broad HeII 44686 line that we observed in C1 (Pakull et al. 2006; Grisé et al., in preparation) provides the decisive proof that this is the optical counterpart of the ULX. Since C1 is a blue stellar object (see Table 5), this result is also in agreement with the typical blue colors found in most other optical ULX counterparts (Goad et al. 2002; Liu et al. 2002, 2004; Kuntz et al. 2005; Liu et al. 2007). In fact, the optical counterpart is expected to contain emission from both the irradiated accretion disk and the donor star. A possible explanation for the prevalence of blue colors was suggested by Madhusudhan et al. (2007) and by Patruno \& Zampieri (2008), based on theoretical evolutionary tracks of X-ray irradiated stars in a binary system.

\subsection{Groups of young stars around X-2}

\subsubsection{Masses and age}

The multicolor image (Fig. 1) already suggests that the stellar population immediately around X-2 is dominated by blue stars, in addition to a more uniform distribution of red, old stars that otherwise dominate the outskirts of the galaxy. The blue stars are largely concentrated in two areas separated by $15^{\prime \prime}$ (See Fig. 1); the cluster located north-east of the ULX appears more rich in stars.

Color-magnitude diagrams (Figs. 4-6) confirm that there are two populations of stars in the field: the old, dominant population of the host galaxy and a blue population with $-0.25 \mathrm{mag}<$ $B-V<0.0$ mag. The brightest members of the young population are at $V \approx 23 \mathrm{mag}$, corresponding to $M_{V} \approx-5 \mathrm{mag}$. We emphasize here that all the bright stars plotted in green in the diagrams (Figs. 4-6) were also detected in the UV band (F330W filter), which confirms that they belong to a young population; the red stars are not visible in the UV band. The brightest blue stars ( $V \lesssim 24 \mathrm{mag}$ ) have probably already left the main sequence, moving along the blue supergiant tracks.

Using isochrones from the Padua (Bertelli et al. 1994; Girardi et al. 2000; Salasnich et al. 2000; Girardi et al. 2002) or Geneva (Lejeune \& Schaerer 2001) evolutionary tracks and taking into account a reddening $E(B-V)=0.1 \mathrm{mag}$ and an extinction based on the Cardelli law (Cardelli et al. 1989), we derive an age for the cluster stars of $\approx 20 \pm 5 \mathrm{Myr}$, which is in disagreement with the value ( $\$ 10 \mathrm{Myr}$ ) reported by Liu et al. (2007) (Sect. 4.5). Our age estimate is based on the most luminous stars that do not suffer from large photometric errors. We used tracks corresponding to a metallicity $Z=0.008$, as suggested by studies of HII regions (Walsh \& Roy 1997; Hadfield \& Crowther 2007); but the inferred ages are only weakly dependent on metallicity. Looking at the evolutionary tracks shown in Fig. 6, we find that the track of a $12 \pm 4 M_{\odot}$ star agrees with the expected distribution of the most luminous blue stars. We conclude that both the mass $\left(12 M_{\odot}\right)$ and brightness $(V \approx-5 \mathrm{mag})$ of these stars are consistent with the inferred age of $\approx 20$ Myr. If on the 

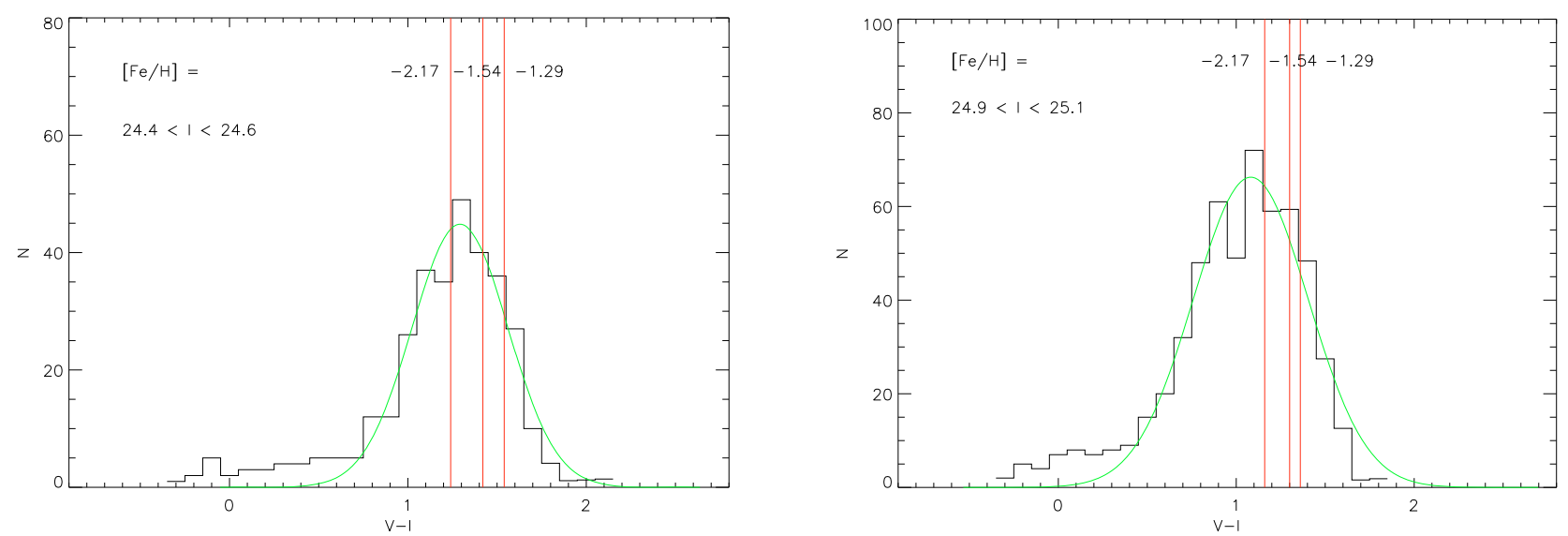

Fig. 3. Color distribution of the stars in our HST/ACS field, at two values of $I$ : 0.5 and 1.0 mag below the TRGB. The vertical lines correspond to the RGB position of three galactic globular clusters at different metallicities.
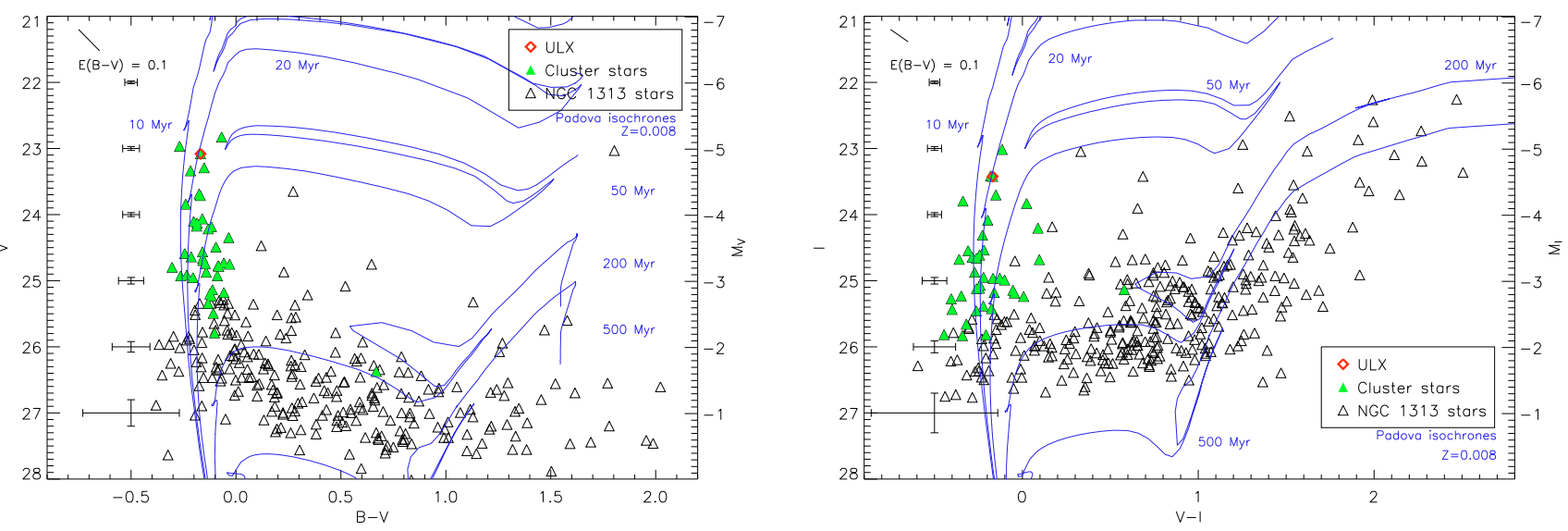

Fig. 4. HST/ACS color-magnitude diagrams for the stellar field around the ULX. HST/ACS magnitudes were transformed into the JohnsonCousins system. Padua isochrones for stars of different ages are overplotted. Typical photometric errors are also plotted. Data have been corrected for the Galactic extinction $(E(B-V)=0.10 \mathrm{mag})$, the bar at the top left corner illustrating this effect. Left panel: color-magnitude diagram in the $(B, V)$ system. Right panel: color-magnitude diagram in the $(V, I)$ system. The same isochrones are plotted in the two panels, i.e. for 10, 20, 50, 200 and $500 \mathrm{Myr}$ at $Z=0.008$. We can see that the two diagrams are largely consistent with each other, excluding the need for a high extragalactic reddening $E(B-V)=0.23$ advocated by Liu et al. (2007). This gives an age of about $20 \mathrm{Myr}$ for the brightest stars in the young cluster.

Table 5. Brightness of the ULX counterpart in different filters, from the HST/ACS observations. Magnitudes are expressed both in the HST/ACS Vegamag system and in the Johnson-Cousins (UBVRI) system, when possible. When converting from $F 555 W$ to $V$ for the 2004 Feb. $22 \mathrm{observation}$, we assumed that the $(B-V)$ color of the counterpart was the same as on 2003 Nov. 22. We also give the brightnesses reported by Liu et al. (2007), that are consistent with our results except for the $F 330 \mathrm{~W} / U$ band.

\begin{tabular}{lcccccc}
\hline \hline Filter & Exposure time (s) & Date & Aperture correction (mag) & VEGAmag & Johnson magnitude & VEGAmag $^{a}$ \\
\hline$F 330 W / U$ & 2760 & 2003 Nov. 22 & 0.885 & $21.733 \pm 0.018$ & $/$ & $22.037 \pm 0.021$ \\
$F 435 W / B$ & 2520 & 2003 Nov. 22 & 0.441 & $23.423 \pm 0.018$ & 23.49 & $23.470 \pm 0.017$ \\
$F 555 W / V$ & 1160 & 2003 Nov. 22 & 0.467 & $23.587 \pm 0.032$ & 23.57 & $23.625 \pm 0.026$ \\
$F 814 W / I$ & 1160 & 2003 Nov. 22 & 0.467 & $23.614 \pm 0.032$ & 23.61 & $23.640 \pm 0.043$ \\
$F 555 W / V$ & 2400 & 2004 Feb. 22 & 0.421 & $23.426 \pm 0.037$ & $\approx 23.41$ & $23.472 \pm 0.021$ \\
\hline
\end{tabular}

${ }^{a}$ These values come from Liu et al. (2007) and are cited for comparison with our work.

other hand we increase the reddening to $E(B-V)=0.20 \mathrm{mag}$ we obtain ages around 10 Myr. However, in this case the two color-magnitude diagrams become less consistent and for a reddening of $E(B-V)=0.30 \mathrm{mag}$, as proposed by Liu et al. (2007), the two diagrams are no longer consistent with each other. In fact, the color-magnitude diagram in the $(B, V)$ system is almost non-physical at this high reddening value because a few bright stars would have a $(B-V)$ color $\lesssim-0.4$ mag. Our independent estimate of the reddening (Sect. 4.5) based on the Balmer decrement of the nebula confirms a low reddening value $(\approx 0.13 \mathrm{mag}$ ) towards $\mathrm{X}-2$.

It may be somewhat surprising that we do not see any red supergiants, which should be present in a 20 Myr-old population, and are often observed in other ULX fields of similar age (e.g. near NGC 4559 X-1, Soria et al. 2005). Evolutionary tracks (e.g. Geneva tracks, Lejeune \& Schaerer 2001) at $Z=0.008$ show that stars in our inferred mass range move to the red part of the diagram for $\approx 0.4 \mathrm{Myr}$, return onto the blue loop for $\approx 1.3 \mathrm{Myr}$ 

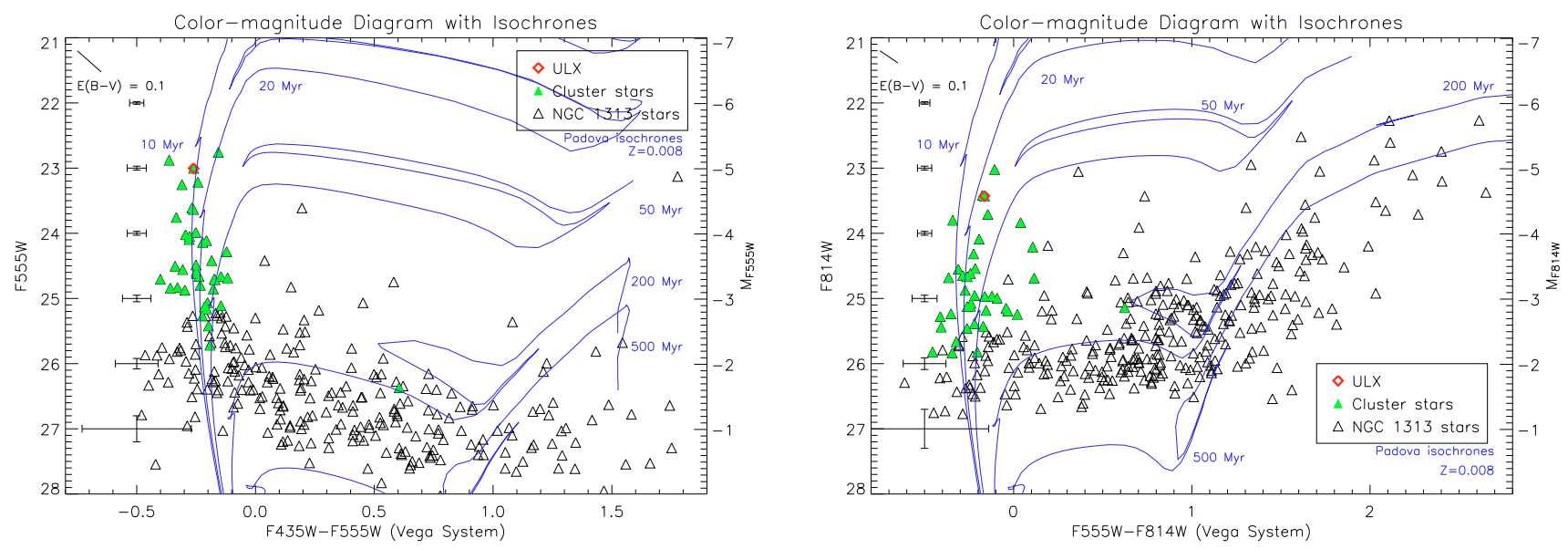

Fig. 5. HST/ACS color-magnitude diagrams in the VEGAMAG photometry system. Left panel: color-magnitude diagram in the (F435W, $F 555 W)$ system. Right panel: color-magnitude diagram in the $(F 555 W, F 814 W)$ system. Our results are mostly consistent with those of Liu et al. (2007); however, we argue that we need a significantly lower extinction than claimed in that paper: $E(B-V)=0.10$ mag instead of $E(B-V)=0.33$ mag which was used to bring the two color-magnitude diagrams into agreement. By comparing these diagrams with those of Fig. 4, we see that the $(V, I)$ and $(F 555 W, F 814 W)$ isochrones are consistent with each other. However, the isochrones in the $(B, V)$ and $(F 435 W$, $F 555 W$ ) systems are shifted by $\approx 0.1 \mathrm{mag}$ in color with respect to each other. We thus find that the Padua isochrones in the $(F 435 W, F 555 W)$ system are different from those in the $(B, V)$ system, transformed with the Sirianni et al. (2005) equations.
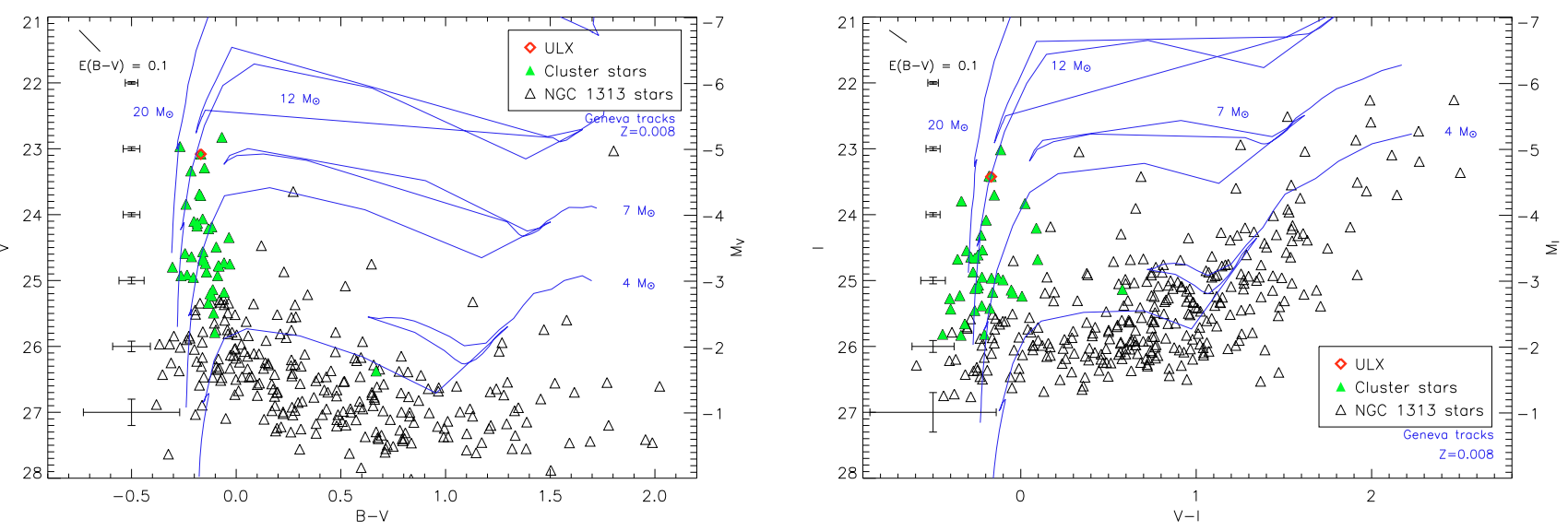

Fig. 6. HST/ACS color-magnitude diagrams (Johnson-Cousins photometry) with Geneva evolutionary tracks for stars of different initial masses $\left(4,7,12\right.$ and $\left.20 M_{\odot}\right)$ with $Z=0.008$. Left panel: color-magnitude diagram in the $(B, V)$ system. Right panel: color-magnitude diagram in the $(V, I)$ system. Both diagrams are consistent with a mass $\approx 12 M_{\odot}$ for the brightest young stars in the field.

and then end up on the red side again for the last $\approx 0.04$ Myr of their lives. We observe 5 stars brighter than $M_{V}=-4.5 \mathrm{mag}$, currently evolving off the main sequence, towards the blue supergiant phase. By taking a blue-to-red supergiant ratio $\sim 3$, a value observed in metal-poor galaxies (Langer \& Maeder 1995), we expect to find about 1 or 2 red supergiants. Given this smallnumber-statistics (coupled with the uncertainty in the duration of the red-supergiant phase), we do not consider the absence of red supergiants in the young cluster to be significant.

\subsubsection{Comparison with $\mathrm{OB}$ associations}

It had been known for some time that ULXs are often associated with galaxy-wide or extended starburst regions (e.g. the Antennae, Zezas et al. 2002). However, some of them, such as Holmberg IX X-1 (Grisé et al. 2006; Ramsey et al. 2006) and the ULX target of this study, seem to be far from any large-scale star-forming activity. But the advent of large optical telescopes and the use of HST have now permitted the detection of small groups of young stars also around these ULXs, strengthening the association of ULXs with young stellar populations. X-2 is far from the center of its host galaxy, in a region where no recent large-scale star-formation episodes have occurred. Only the two young groups of stars described above are present, superimposed on a predominantly old- and intermediate-age population. These young groups are certainly not gravitationally bound, because their density is too low (some tens of stars scattered over $200 \mathrm{pc}$ ). Based on their integrated luminosities, we infer from Starburst99 simulations (Leitherer et al. 1999) that the two young stellar associations have masses $M \approx(5 \pm 1) \times 10^{3} M_{\odot}$ for the north-west group and $M \approx(1.5 \pm 0.5) \times 10^{3} M_{\odot}$ for the south-east group. So, they have similar masses and sizes as typical OB associations in our Galaxy and other Local Group galaxies $\left(\sim 10^{3} M_{\odot}\right.$ in $\approx 200$ pc, e.g. Gouliermis et al. 2003$)$. The obvious question is what triggered this recent, isolated episode of star formation in this outer part of the galaxy? It was suggested (e.g. Soria 2006) that several ULXs are located in regions perturbed by tidal interactions or collisions. An intriguing, unexplained feature of NGC 1313 (in particular, of its southern half) is the presence of isolated HII regions (located $\approx 3^{\prime}$ north of the ULX), and of some unusual, expanding HI supershells. 

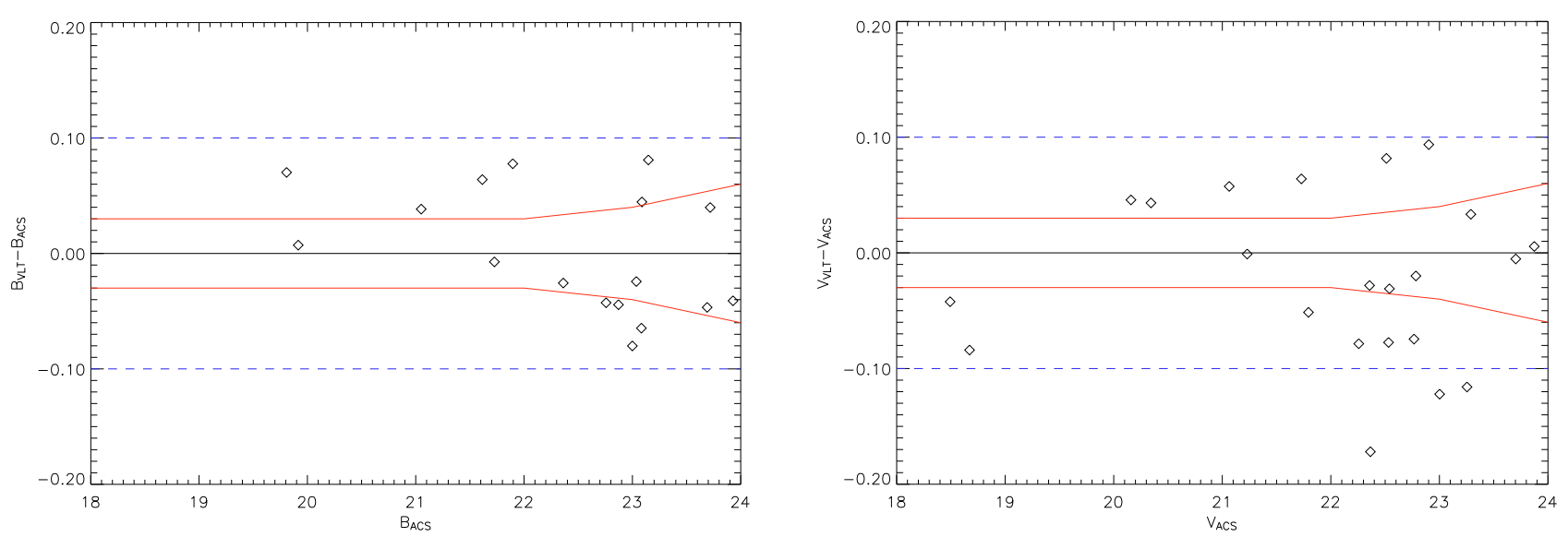

Fig. 7. Difference between VLT/FORS1 and HST/ACS photometry, in the $B$ (left panel) and $V$ band (right panel). For this comparison, we only used bright, isolated stars. We find that $40 \%$ of them lie within the combined photometric errors of the HST and VLT observations (solid red lines), and for most of the others the difference is only $\leq 0.1 \mathrm{mag}$ (dashed blue lines).

Table 6. Brightness of the ULX counterpart in different filters, from our VLT/FORS1 observations. To minimize the effects of variability, the $B-V$ color was estimated using the 2003 Dec. $25 B$ exposure, while the $B-R$ color was estimated using the 2003 Dec. $24 B$ exposure.

\begin{tabular}{lcccc}
\hline \hline Filter & Exposure time (s) & Date & Magnitude & Absolute magnitude \\
\hline$B$ & $420 / 840$ & 2003 Dec. 24/25 & $23.40 \pm 0.02 / 23.44 \pm 0.03$ & -5.0 \\
$V$ & 600 & 2003 Dec. 25 & $23.45 \pm 0.04$ & -4.9 \\
$R$ & 500 & 2003 Dec. 24 & $23.58 \pm 0.05$ & -4.6 \\
\hline
\end{tabular}

It was previously suggested (Sandage \& Brucato 1979; Blackman 1981; Peters et al. 1994) that the southern side of the galaxy has been affected by a collision or tidal interaction with a satellite galaxy. However, it was also noted (Ryder et al. 1995) that the largest HI supershell is spatially associated with the southern HII regions. Thus, it is also possible that the isolated HII regions and other local episodes of star formation are the result of collisions of large HI clouds with the galactic disk (Marcelin \& Gondoin 1983). It was recently noticed that such phenomena may be associated with localized star formation in NGC 4395 (Heald \& Oosterloo 2007). We speculate that the isolated complex of young stars around the ULX may also have been formed through a similar event.

\subsection{Comparison between HST and VLT photometry}

One of the objectives of our study is to see whether there have been significant changes in the brightness of the ULX counterpart between the HST and VLT observations. To do so, we need to compare its brightness with those of neighboring, isolated stars in the two observations. This will also provide a check on the absolute photometric calibration between the two datasets. Such a comparison is partly hampered by the difference in resolution: most of the stars detected as single sources in the VLT images are resolved into multiple components in the HST images. Besides, the brightest stars are usually saturated in one or the other datasets.

Nonetheless, there are about twenty bright, isolated sources that appear point-like and not saturated in either of the VLT and HST images. Crucially, the seeing of the VLT observations was good enough (Table 2) to resolve the true ULX counterpart from its close companion. In Fig. 7 we show the magnitude difference of these stars observed with FORS 1 and ACS $B$ and $V$ filters. For $\approx 40 \%$ of these stars the HST and VLT brightnesses are the same within the photometric errors of the two observations. But even for the other stars, the discrepancy does not exceed $\approx 0.1 \mathrm{mag}$ in either filter. Overall, the standard $1 \sigma$ deviations are $\sigma_{B}=0.05 \mathrm{mag}$ and $\sigma_{V}=0.07 \mathrm{mag}$. We conclude that absolute photometry between VLT and HST can be achieved to a precision of less than about 0.1 mag.

We have then carried out a photometric study of the ULX counterpart in the VLT/FORS 1 images (Table 6). Its colors could be affected by an additional error of $\approx 0.1$ mag due to variability in one of the filters between different observations. As we do not have consecutive frames in different filters, we used the closest observations available to minimize the effect of color variability. Once again, we can see that the absolute brightness measured in the VLT frames is in good agreement with the HST results. We will later show (Sect. 5.2) that the remaining differences could be explained as intrinsic variability of the ULX counterpart.

\subsection{On the conversion between the ACS photometric system and more standard colors}

The HST/ACS data of X-2 have also been analyzed and discussed in two recent papers (Ramsey et al. 2006; Liu et al. 2007). We have carefully retraced their analyses and noticed some differences with respect to the results we have independently obtained from the same dataset. Most of the discrepancy seems to be related to the choice of photometric system for the datapoints and the evolutionary tracks employed.

We analyzed the data in two different ways which, in principle, should lead to identical conclusions. First, we compared the datapoints directly measured in the HST/ACS system colors with the Padua isochrones in the HST/ACS system. Then, we transformed the HST/ACS colors to the more-standard Johnson-Cousins colors via the Sirianni et al. (2005) transformations, and compared these datapoints with the Padua JohnsonCousins isochrones. Both sets of isochrones were independently 
calculated and supplied by Girardi et al. (2002) and Girardi ${ }^{1}$ (in preparation).

Surprisingly these two methods give different results (Figs. 4 and 5), leading to different estimates of stellar ages and masses! To investigate the reason for this discrepancy, we also transformed the Padua Johnson-Cousins isochrones to the HST/ACS system using the recommended Sirianni et al. (2005) transformations: the result is different from the Padua HST/ACS isochrones supplied by Girardi (in preparation), especially for the $B$ band.

Comparing our results with those of Liu et al. (2007), we can immediately see the effect of this discrepancy. Although our observed brightnesses and colors are consistent with theirs (Table 5), our determination of the age of the young stellar population is different ( $\approx 20 \mathrm{Myr}$ instead of their $\lesssim 10 \mathrm{Myr}$ ), with obvious consequences for the physical interpretation of the ULX. The Padua HST/ACS isochrones suggest different ages of the young stars in the ( $F 555 W$ versus $F 435 W-F 555 W)$ and $(F 814 W$ versus $F 555 W-F 814 W$ ) color-magnitude diagrams (Fig. 5). An obvious way to reconcile this discrepancy is to take the younger age and assume a high local extinction, which led Liu et al. (2007) to conclude that $E(B-V)=0.33 \mathrm{mag}$.

However, as we showed in Sect. 4.3.1, if we transform the observed brightnesses and colors to the Johnson-Cousins system and compare them with the Padua Johnson-Cousins isochrones, we obtain the same age in the ( $V$ versus $B-V$ ) and ( $I$ versus $V-I$ ) color-magnitude diagrams (Fig. 4) with no need for an extinction greater than the Galactic line-of-sight value. An independent argument in favor of low extinction comes from the spectrum of the bubble nebula around the ULX, in which the Balmer decrement suggests $E(B-V)=0.13 \pm 0.03$ mag (Grisé et al., in preparation), which is inconsistent with the higher values advocated by Liu et al. (2007). Thus, we suspect that the Padua Johnson-Cousins isochrones are the more reliable set of tracks. In any case, we emphasize that the discrepancy between the two widely-used sets of tracks in the literature deserves further investigation. We caution that conclusions based on ACS F435W and $F 555 \mathrm{~W}$ photometry alone and in combination with corresponding Padua evolutionary tracks may well be misleading.

The $F 435 W$ and $F 555 W$ filter data from the same HST/ACS dataset were also studied by Ramsey et al. (2006). They derived an age $\gtrsim 10^{7} \mathrm{yr}$ and stellar masses $\lesssim 10 \mathrm{M}_{\odot}$. Although their results appear to be in agreement with ours, we cannot directly compare our analysis with their approach, because their paper does not provide information on how they converted from $F 435 W$ and $F 555 W$ to $B$ and $V$ bands. Moreover, there seem to be some inconsistencies because the galactic extinction is not accounted for when they estimate the stellar age. Finally, Ramsey et al. (2006) found that the ULX counterpart has $M_{V}=-3.96 \pm 0.02 \mathrm{mag}$, which is clearly inconsistent (by $\approx 1.0 \mathrm{mag}$ ) with both our result and that of Liu et al. (2007).

\section{Nature of the ULX optical counterpart}

\subsection{Constraints from photometry}

We have seen (Sect. 4.3.1) that the young population of stars is consistent with an age of $(20 \pm 5) \mathrm{Myr}$, and with an upper mass limit for non-collapsed stars of $(12 \pm 4) M_{\odot}$. Although it is problematic to draw any inferences about the ULX mass-donor star from photometry alone, due to unknown effects of X-ray irradiation and binary evolution (Patruno \& Zampieri 2008), we can

\footnotetext{
1 See http://pleiadi.pd.astro.it and http://stev.oapd. inaf.it/cmd for data access
}

at least say that the ULX optical counterpart shares the same brightness and colors as the brightest stars in the young association. If the counterpart is believed to be optically dominated by a normal star, its magnitude $\left(M_{V} \sim-5 \mathrm{mag}\right)$ is consistent with those of an early B-type or a late-type $\mathrm{O}$ star, although its colors $\left((B-V)_{0} \sim-0.18,(V-I)_{0} \sim-0.16\right)$ are more consistent with an already somewhat evolved B-type star. But taking into account that we also expect a contribution from the accretion disk to the integrated properties of the ULX counterpart (see Sect. 5.2), the estimated values of brightness and hence mass for the donor star are necessarily upper limits. In fact, numerical models of stellar-mass black-hole X-ray binaries with donor stars in the mass range $2-17 M_{\odot}$ (Rappaport et al. 2005) suggest that the accretion disk should dominate the optical emission from the binary system.

From the empirical point of view, we draw attention to the well-known van Paradijs \& McClintock (1994) diagram which shows a strong correlation over 10 absolute magnitudes between the observed quantity $\Sigma$ and the absolute visual magnitude $M_{V}$ for disk dominated low mass X-ray binaries. Here, $\Sigma=\left(L_{\mathrm{X}} / 10^{38} \mathrm{erg} / \mathrm{s}\right)^{1 / 2} \times(P / 1 \mathrm{~h})^{2 / 3} \times\left(M /\left(2 M_{\odot}\right)\right)^{1 / 3}$ which reflects the simple assumption that the total bolometric luminosity $L_{\text {bol }}$ of an X-ray irradiated disk is proportional to the X-ray luminosity and the disk area. At optical wavelengths, the surface brightness varies as $T^{2}$, hence $L_{\mathrm{opt}} \sim L_{\mathrm{bol}}^{1 / 2} \times a$, where a is the orbital separation. Following black hole binary evolutionary model computations by Podsiadlowski et al. (2003) and Rappaport et al. (2005), an orbital period of 1-6 days and a total mass of some $20 M_{\odot}$ are expected for the ULX which, using $L_{\mathrm{X}} \sim 10^{40} \mathrm{erg} / \mathrm{s}$ results in $\log \Sigma=2.2-2.7$. In the van Paradijs \& McClintock (1994) diagram such values correspond to an absolute disk magnitude $M_{V}=-4.0--5.0$ which in fact is close to the observed brightness of the optical counterpart of X-2.

Taking the likely disk contribution into account, we conclude that the donor star in the ULX system has a mass of $\approx 10 M_{\odot}$ (Rappaport et al. 2005).

With the currently available data, it is however difficult to make more specific statements about the companion star. More information will come from our spectroscopic study currently in progress $^{2}$, which will constrain the orbital period and hence the size of the system, and will help in resolving the stellar and disk contribution to the optical spectrum. Deep spectra will possibly identify narrow absorption lines from the donor star as well as broader emission lines from the disk.

\subsection{Photometric variability in the VLT data}

In addition to determining average brightnesses and colours, we have searched for short and long term variability of the ULX counterpart. To that end we have applied differential photometry by using four relatively bright ( $B \sim 19-20 \mathrm{mag}$ ) comparison stars with relative photometric errors $<0.03$ mag. We have plotted (Fig. 8, left panel) the $B$-band light curve of the counterpart and of a slightly fainter comparison (check) star of similar brightness. Each datapoint refers to an 840-s observation, which can be either a single exposure or two consecutive 420-s exposures, depending on the observational setup. The observing conditions were clear with good (sub-arcsec) seeing (Table 2). The error bars are relatively large because of the relatively large airmasses in many of our exposures; NGC 1313 is low in the Paranal sky (airmass $\gtrsim 1.3$ ).

\footnotetext{
$213 \mathrm{~h}$ of observations executed on VLT/FORS1.
} 

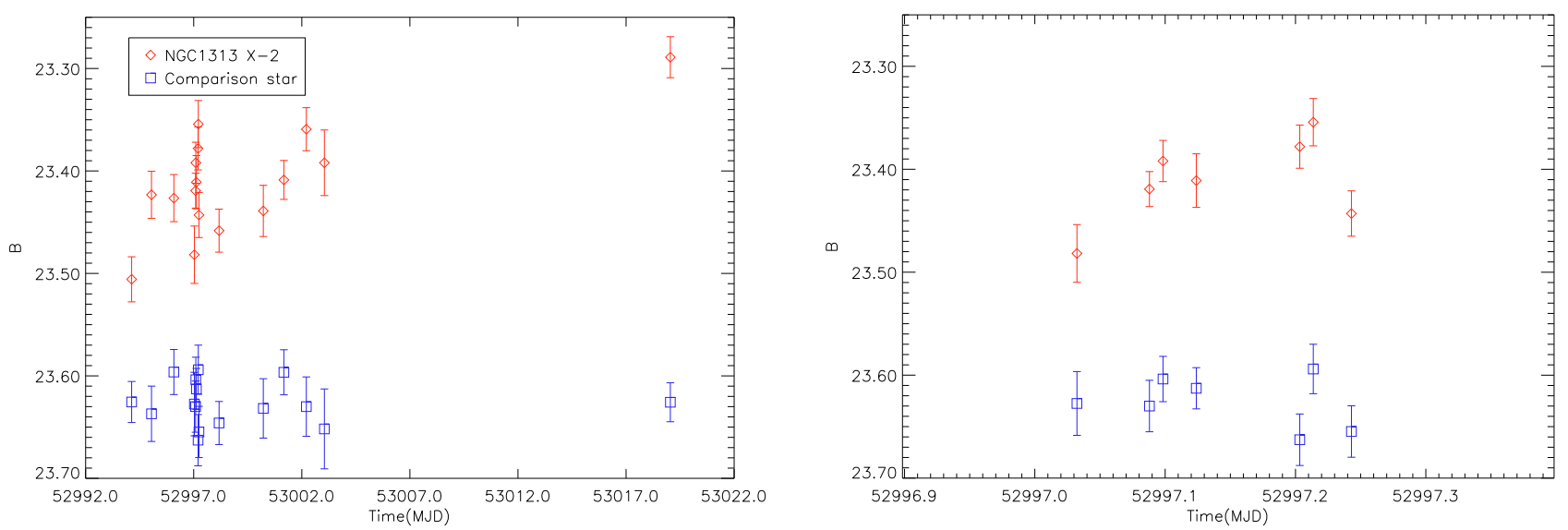

Fig. 8. Left panel: $B$-band variability of the ULX counterpart (red datapoints) over 1 month of observations with VLT/FORS1. The blue datapoints show the much less variable behavior of a comparison star of similar brightness and color; its zeropoint has been shifted downwards by 0.22 mag for clarity. Right panel: zoomed-in view of the ULX brightness during the 2003 December 24 observations: the source (red datapoints) shows $\approx 0.10$ mag variability on timescales of about one hour. See Sect. 5.2 for a discussion on the statistical significance of such variability.

First, we found a trend of increasing brightness for the ULX counterpart (Fig. 8), with a change of $\approx 0.2$ mag over the whole observing period. Moreover, repeated observations during the night of December 24 show the ULX counterpart to also be variable on timescales of about one hour. By contrast, the comparison star shows significantly less scatter, and is consistent with having a constant brightness within the photometric errors. The maximum amplitude of the variations is $0.22 \pm 0.04 \mathrm{mag}$ for the ULX counterpart, and only $0.06 \pm 0.05$ mag for the comparison star. We used a $\chi^{2}$ test to assess more quantitatively whether or not the observed variability is statistically significant. To do this, we calculated

$\chi^{2} \equiv \sum_{i=1}^{n} \frac{\left(x_{i}-\bar{x}\right)^{2}}{\sigma_{i}^{2}}$,

where $n$ is the number of observations (with $n-1$ degrees of freedom), $x_{i}$ are the measured brightnesses, $\bar{x}$ the median brightness and $\sigma_{i}$ the error on each measure. Here we multiplied the formal error given by DAOPHOT by 1.15 in order to match the error distribution of the stars in the field with the expected $\chi^{2}$ distribution in Fig. 9. For the ULX counterpart, we obtain $\chi^{2}=69.0$ for 15 d.o.f., corresponding to a probability of only $7 \times 10^{-9}$ for the null hypothesis of constant brightness. For the comparison star we obtain $\chi^{2}=9.7$ for 15 d.o.f., corresponding to a constantbrightness probability of $84 \%$. During the night of December 24, we find $\chi^{2}=13.6$ for 6 d.o.f. for the counterpart which translates into a probability of $3.4 \%$ for it being constant. The comparison star has $\chi^{2}=5.2$ for 6 d.o.f., which gives a probability of $52 \%$ for the null hypothesis.

Thus, we have clear evidence that the ULX counterpart is variable on timescales ranging from hours to several days during our VLT observations. We further tested the significance of this variability by comparing the behavior of all the point-like sources in the brightness range $23 \mathrm{mag}<B<24 \mathrm{mag}$. For 92 out of 109 sources (84\%), we obtain a constant-brightness probability of more than $5 \%$, as expected from non-variable sources (Fig. 9). The ULX counterpart clearly shows much higher scatter in its brightness measurements, compared with most other stars. Only $2 \%$ of the field stars display variability equal or greater than the ULX counterpart. In fact, a careful inspection of the position of the stars on the frame shows that the great majority of stars with a probability below $0.1 \%$ for the null hypothesis of constant brightness are located in the north-west sector, where crowding

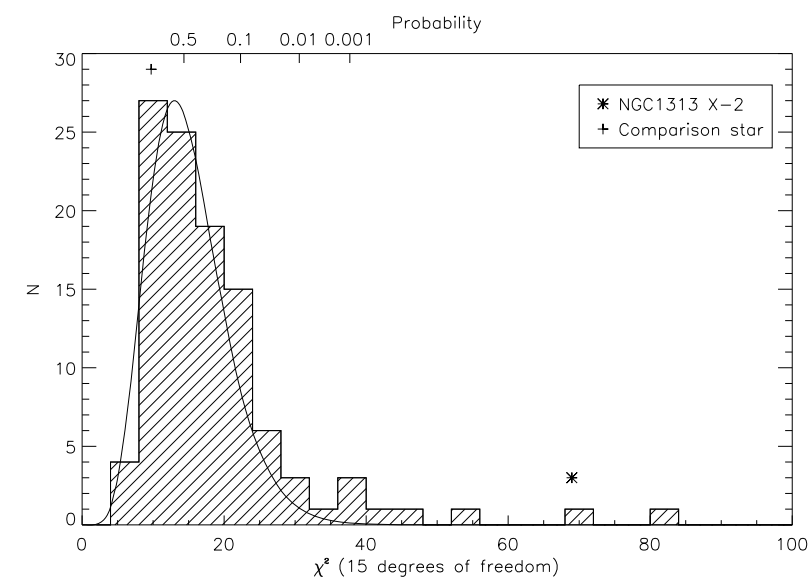

Fig. 9. $\chi^{2}$ test of the null hypothesis (no brightness variability) for all the stars in the VLT/FORS1 frame with 23 mag $<B<24$ mag. With a $\chi^{2} \approx 84.97$ for 15 degrees of freedom (i.e., a probability of constant brightness of $7 \times 10^{-9}$ ), the ULX counterpart is more variable than almost all the other sources in the field, including the star we chose for comparison in Fig. 8. Overplotted is the $\chi^{2}$ distribution for 15 degrees of freedom.

is more severe, or are situated in the immediate vicinity of other bright stars. In those cases, a very small fluctuation of the seeing may severely affect PSF fitting and brightness estimates. These stars were removed from the sample and are therefore not plotted in Fig. 9. Note that the ULX counterpart does not suffer from confusion with any other nearby sources. In conclusion, we argue that the observed variability of the ULX counterpart is statistically significant and is not due to spurious effects such as source confusion.

We believe that the observed scatter in the ULX brightness is a real effect, probably due to the same kind of short-term variability seen in many low-mass X-ray binaries (for example, in LMC X-2: McGowan et al. 2003). This is generally understood as disk reprocessing of variable $\mathrm{X}$-ray emission although simultaneous optical and X-ray observations do not always show a clear correlation between the brightnesses in these bands.

Other luminous stars are also known to display irregular $\sim 0.2$ mag variations in their light curves: in particular, Be stars (e.g. Dachs 1987), which would also have a luminosity consistent with this ULX counterpart. In Be stars, the variability is 
mostly due to the formation of a circumstellar disk or extended envelope, with typical radii of a few times the stellar radius (e.g. Porter \& Rivinius 2003). However, the donor star in a ULX is thought to be almost persistently filling its Roche lobe; accretion onto the $\mathrm{BH}$ from a stellar wind or a Be disk is not sufficient to produce the observed, persistent X-ray luminosity and would probably give rise to large X-ray outbursts.

In any case, the optical variability suggests a non-negligible flux contribution from the accretion disk - unlike the situation encountered in normal high-mass X-ray binaries, where the optical light is dominated by emission from the OB mass donor.

In order to look for periodic variations we computed a LombScargle periodogram of the light curve (IDL scargle routine ${ }^{3}$, Joern Wilms 2000); however, no significant period was found. This indicates that we are not primarily seeing ellipsoidal variations of the companion star over the binary orbit as would be expected by a Roche-lobe filling star not seen pole-on. However, such variations could be masked by the random variations of the reprocessed X-ray flux on the accretion disk and on the irradiated hemisphere of the companion.

In view of this interpretation, it is useful to compare the optical and X-ray variability. X-2 was observed by XMM-Newton at the same time as the VLT observation. It was found (Mucciarelli et al. 2007) that the X-ray brightness rose sharply between 2003 Dec. 21 and 25 (resp. MJD 52294 and MJD 52 998) and declined again afterwards. By contrast, we do not see a corresponding optical flare on a similar timescale (Fig. 11). The optical counterpart did not become fainter after the end of the X-ray flare; instead, it was $\approx 0.2$ mag brighter on 2004 Jan. 15 than during 2003 Dec. 24-25, even though the X-ray luminosity was lower by a factor 2 .

\subsection{Variability in the combined VLT/HST data set}

We showed in Sect. 4.4 that the absolute photometry of our HST and VLT observations are consistent with each other. Therefore, we can extend our variability study by adding the two HST/ACS datapoints to the $B$-band VLT light curve discussed earlier. We do not have an F435W HST/ACS observation on 2004 Feb. 22, but we have used the $F 555 W$ brightness and converted it to a $B$ brightness by using the $B-V$ color measured in the 2003 Nov. HST data. Assuming that the color has stayed constant, we find that the optical counterpart has brightened by $\approx 0.15$ mag with respect to the first HST observation three months earlier (Fig. 10).

\section{Conclusions}

We combined the high sensitivity of VLT/FORS1 and the high spatial resolution of $\mathrm{HST} / \mathrm{ACS}$ for a detailed photometric study of the optical counterpart to the ULX X-2 and of its immediate environment in the southern part of the galaxy NGC 1313. The dominant stellar population in that region of the galaxy is old ( $>1$ Gyr). From the brightness and colours of its red giant branch, we re-estimated the distance to the galaxy as $4.07 \pm$ $0.22 \mathrm{Mpc}$, consistent with previous studies which used observations from the north-west part of the galaxy. We also estimated that the average metallicity of the old stellar population is $[\mathrm{Fe} / \mathrm{H}]=-1.9 \pm 0.3$, consistent with the typical metallicity found in haloes of spiral galaxies of similar size.

\footnotetext{
${ }^{3}$ http://astro.uni-tuebingen.de/software/idl/aitlib/ timing/scargle.html
}

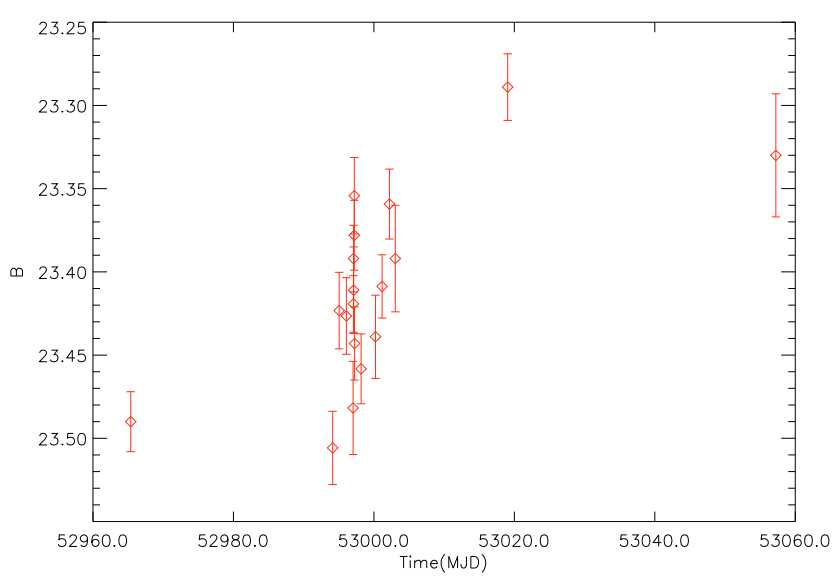

Fig. 10. Combined $B$-band light curve of the ULX counterpart between 2003 November 22 and 2004 February 22, from the VLT/FORS1 and HST/ACS data. The HST/ACS datapoints are the first and last one.

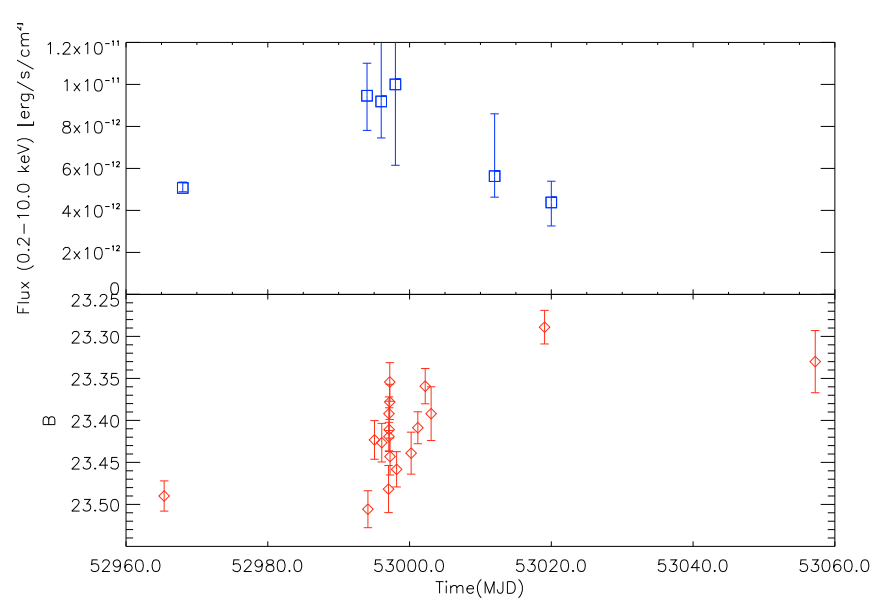

Fig. 11. X-ray (top) and B-band (bottom) light curve of the ULX counterpart. Note that the X-ray flux is taken from Mucciarelli et al. (2007).

Near the ULX, we highlighted two groups of (a few) young stars, spread out over $\approx 200 \mathrm{pc}$, and hence more similar to an OB association (or more likely, two separate associations close to each other) rather than to a bound cluster. They clearly stand out in brightness and colors over the surrounding old population. There are no other similar groups of young stars in this region of the galaxy, nor are they connected to spiral arm features. The reason for this recent, localized episode of star formation is unclear, but the ULX is clearly associated with this young population. We speculate that the local star-formation episode may have been triggered by a collisional event (proposed for the Gould's Belt in the Milky Way) with a satellite galaxy or, more likely, with a fast-moving HI cloud transiting across the disk plane (for which there is independent evidence in NGC 1313).

We estimate that the largest association of young stars has an age $\approx 20 \mathrm{Myr}$ and a stellar mass $\approx 5 \times 10^{3} M_{\odot}$. The ULX optical counterpart appears as one of the brightest stars in the association, without any obvious color or brightness anomaly. Using standard stellar evolutionary tracks, we constrain its mass to be $\lesssim 12 M_{\odot}$; or even less, if the accretion disk is significantly contributing to the source luminosity. Our mass estimate is smaller than reported in previous work (Liu et al. 2007), and correspondingly, we estimate relatively older ages for the young stars. We argue that the most likely reason for that is a discrepancy between the Padua isochrones in the HST/ACS 
VEGAMAG system and those in the standard Johnson-Cousins system. The two sets of isochrones are not related to each other via the same Sirianni transformations (Sirianni et al. 2005) that are generally accepted as the best way to transform the brightness and colors of the observed sources from the HST/ACS VEGAMAG system to the Johnson-Cousins system. We showed that the HST/ACS isochrones indicate different ages for the same dataset, in the $(F 814 W, F 555 W-F 814 W)$ and $(F 555, F 435-F 555 W)$ color-magnitude diagrams. By contrast, the Johnson-Cousins isochrones give consistent ages, when applied to the same datapoints transformed via Sirianni's equations. For this reason, we believe that the Johnson-Cousins isochrones are more reliable. In any case, this is an issue deserving further investigation. Although the resulting differences in masses and ages may appear small, they can have a large effect on the physical interpretation of the ULX, for example regarding its accretion rate and duration of the active phase.

One of the most significant findings of this work is the shortterm variability of the ULX counterpart, by up to $\approx 0.2 \mathrm{mag}$, on timescales of hours and days. This is detected both in the HST/ACS and in the VLT/FORS1 datasets, and even more evident in the combined dataset. There is no evidence of periodicity. This suggests that the variability is not due to ellipsoidal variations. Instead, it may be caused by varying X-ray irradiation of the donor star and (more likely) a stochastically-varying contribution from the accretion disk.

Our work, and other recent multi-band studies of ULXs, suggest that those systems share most of their properties with Galactic X-ray binaries, although at higher luminosities. The donor star need not be an extraordinarily massive object, or even an $\mathrm{O}$ star; in fact, it appears to be a common-or-garden B star. The accretion disk may be as luminous (or possibly even more luminous) as the donor star, even in the optical bands, which is consistent with a high X-ray luminosity and high accretion rate. We also showed that the total stellar mass in the young stellar associations around the X-2 ULX is $<10^{4} M_{\odot}$ : thus X-2 is clearly not in a super-star-cluster. This means that whatever the nature of the compact object is (stellar-mass or IMBH), it was not formed via a runaway-coalescence scenario as proposed by Ebisuzaki et al. (2001) and Portegies Zwart \& McMillan (2002). But X-2 has been proposed as one of the best candidates for an IMBH $\left(M \sim 1000 M_{\odot}\right)$, because this ULX has a supersoft spectral component at $0.15 \mathrm{keV}$ (Miller et al. 2003). We have shown in our study that X-2 sits in a normal OB association so if this ULX is an IMBH, it means that there must be other ways of forming such objects. More likely, we suggest that X-2 is not an IMBH and that it was formed within the stellar association via a more conventional stellar evolutionary scenario. We point out here that X-2 is not an exceptional case. Indeed, most other luminous ULXs do not sit in super-star-clusters either (e.g. Holmberg IX X-1), but are part of much smaller associations. In those cases, either there is a different way to form IMBHs, or more likely most of them have a mass $\lesssim 100 M_{\odot}$ and formed from ordinary stellar evolution.

For a definitive answer on the $\mathrm{BH}$ mass it is crucial to derive the parameters of the binary system, via phase-resolved observations. The apparently random variability of the optical counterpart, interpreted as the effect of X-ray reprocessing in the accretion disk and on the surface of the secondary will probably mask periodic variability such as ellipsoidal variations or the X-ray heating curve. Thus, it may be hard to detect the signature of the binary period from photometric observations. On the other hand, spectroscopic observations (currently in progress) may allow us to determine the phase-resolved radial-velocity curve of the HeII $\lambda 4686$ disk emission line, and hence to constrain the BH mass.

Based on observations made with ESO Telescopes at the Paranal Observatory under programme ID 072.D0614 and on observations made with the NASA/ESA Hubble Space Telescope, obtained from the data archive at the Space Telescope Institute. STScI is operated by the association of Universities for Research in Astronomy, Inc. under the NASA contract NAS 5-26555.

\section{References}

Abolmasov, P., Fabrika, S., Sholukhova, O., \& Afanasiev, V. 2007a, Astrophys. Bull., 62, 36

Abolmasov, P. K., Swartz, D. A., Fabrika, S., et al. 2007b, ApJ, 668, 124 Begelman, M. C. 2002, ApJ, 568, L97

Bertelli, G., Bressan, A., Chiosi, C., Fagotto, F., \& Nasi, E. 1994, A\&AS, 106, 275

Bertin, E., \& Arnouts, S. 1996, A\&AS, 117, 393

Blackman, C. P. 1981, MNRAS, 195, 451

Cardelli, J. A., Clayton, G. C., \& Mathis, J. S. 1989, ApJ, 345, 245

Colbert, E. J. M., \& Mushotzky, R. F. 1999, ApJ, 519, 89

Da Costa, G. S., \& Armandroff, T. E. 1990, AJ, 100, 162

Dachs, J. 1987, in Physics of Be Stars, ed. A. Slettebak, \& T. P. Snow, IAU Colloq., 92, 149

de Vaucouleurs, G., de Vaucouleurs, A., Corwin, Jr., H. G., et al. 1991, Third Reference Catalogue of Bright Galaxies (Berlin, Heidelberg, New York: Springer-Verlag), 1-3, 2069

Dewangan, G. C., Griffiths, R. E., \& Rao, A. R. 2006, ApJ, 641, L125

Ebisuzaki, T., Makino, J., Tsuru, T. G., et al. 2001, ApJ, 562, L19

Fabbiano, G., Zezas, A., King, A. R., et al. 2003, ApJ, 584, L5

Feng, H., \& Kaaret, P. 2006, ApJ, 650, L75

Girardi, L., Bressan, A., Bertelli, G., \& Chiosi, C. 2000, A\&AS, 141, 371

Girardi, L., Bertelli, G., Bressan, A., et al. 2002, A\&A, 391, 195

Goad, M. R., Roberts, T. P., Knigge, C., \& Lira, P. 2002, MNRAS, 335, L67

Gouliermis, D., Kontizas, M., Kontizas, E., \& Korakitis, R. 2003, A\&A, 405, 111

Grisé, F., Pakull, M. W., \& Motch, C. 2006, in Populations of High Energy Sources in Galaxies, ed. E. J. A. Meurs, \& G. Fabbiano, IAU Symp., 230, 302

Hadfield, L. J., \& Crowther, P. A. 2007, MNRAS, 381, 418

Heald, G., \& Oosterloo, T. 2007 [arXiv: 0712.1184]

Irwin, J. A., Bregman, J. N., \& Athey, A. E. 2004, ApJ, 601, L143

Kaaret, P., Ward, M. J., \& Zezas, A. 2004, MNRAS, 351, L83

Karachentsev, I. D., Karachentseva, V. E., Huchtmeier, W. K., \& Makarov, D. I. 2004, AJ, 127, 2031

King, A. R., Davies, M. B., Ward, M. J., Fabbiano, G., \& Elvis, M. 2001, ApJ, 552, L109

Koekemoer, A. M., Fruchter, A. S., Hook, R. N., \& Hack, W. 2002, in The 2002 HST Calibration Workshop: Hubble after the Installation of the ACS and the NICMOS Cooling System, ed. S. Arribas, A. Koekemoer, \& B. Whitmore, 337

Krauss, M. I., Kilgard, R. E., Garcia, M. R., Roberts, T. P., \& Prestwich, A. H. 2005, ApJ, 630, 228

Kuntz, K. D., Gruendl, R. A., Chu, Y.-H., et al. 2005, ApJ, 620, L31

La Parola, V., Peres, G., Fabbiano, G., Kim, D. W., \& Bocchino, F. 2001, ApJ, 556,47

Lang, C. C., Kaaret, P., Corbel, S., \& Mercer, A. 2007, ApJ, 666, 79

Langer, N., \& Maeder, A. 1995, A\&A, 295, 685

Lee, M. G., Freedman, W. L., \& Madore, B. F. 1993, ApJ, 417, 553

Leitherer, C., Schaerer, D., Goldader, J. D., et al. 1999, ApJS, 123, 3

Lejeune, T., \& Schaerer, D. 2001, A\&A, 366, 538

Liu, J.-F., Bregman, J. N., \& Seitzer, P. 2002, ApJ, 580, L31

Liu, J.-F., Bregman, J. N., \& Seitzer, P. 2004, ApJ, 602, 249

Liu, J.-F., Bregman, J., Miller, J., \& Kaaret, P. 2007, ApJ, 661, 165

Madhusudhan, N., Rappaport, S., Podsiadlowski, P., \& Nelson, L. 2007 [arXiv: 0710.3854]

Makishima, K., Kubota, A., Mizuno, T., et al. 2000, ApJ, 535, 632

Marcelin, M., \& Gondoin, P. 1983, A\&AS, 51, 353

McGowan, K. E., Charles, P. A., O’Donoghue, D., \& Smale, A. P. 2003, MNRAS, 345, 1039

McHardy, I. M., Koerding, E., Knigge, C., Uttley, P., \& Fender, R. P. 2006, Nature, 444, 730

Méndez, B., Davis, M., Moustakas, J., et al. 2002, AJ, 124, 213

Miller, J. M., Fabbiano, G., Miller, M. C., \& Fabian, A. C. 2003, ApJ, 585, L37 
Miller, N. A., Mushotzky, R. F. \& Neff, S. G. 2005, ApJ, 623, L109 Mizuno, T., Miyawaki, R., Ebisawa, K., et al. 2007, PASJ, 59, 257

Mouhcine, M., Ferguson, H. C., Rich, R. M., Brown, T. M., \& Smith, T. E. 2005, ApJ, 633, 821

Mucciarelli, P., Zampieri, L., Falomo, R., Turolla, R., \& Treves, A. 2005, ApJ, 633, L101

Mucciarelli, P., Casella, P., Belloni, T., Zampieri, L., \& Ranalli, P. 2006, MNRAS, 365, 1123

Mucciarelli, P., Zampieri, L., Treves, A., Turolla, R., \& Falomo, R. 2007, ApJ, 658, 999

Pakull, M. W., \& Grisé, F. 2008 [arXiv: 0803. 4345]

Pakull, M. W., Grisé, F., \& Motch, C. 2006, in Populations of High Energy Sources in Galaxies, ed. E. J. A. Meurs, \& G. Fabbiano, IAU Symp., 230, 293

Pakull, M. W., \& Mirioni, L. 2002 [arXiv: astro-ph/0202488]

Pakull, M. W., \& Mirioni, L. 2003, in Rev. Mex. Astron. Astrofis. Conf. Ser., 15, ed. J. Arthur, \& W. J. Henney, 197

Patruno, A., \& Zampieri, L. 2008, MNRAS, 386, 543

Pellerin, A., Meyer, M., Harris, J., \& Calzetti, D. 2007, ApJ, 658, L87

Peters, W. L., Freeman, K. C., Forster, J. R., Manchester, R. N., \& Ables, J. G. 1994, MNRAS, 269, 1025

Podsiadlowski, P., Rappaport, S., \& Han, Z. 2003, MNRAS, 341, 385

Portegies Zwart, S. F., \& McMillan, S. L. W. 2002, ApJ, 576, 899

Porter, J. M., \& Rivinius, T. 2003, PASP, 115, 1153

Ramsey, C. J., Williams, R. M., Gruendl, R. A., et al. 2006, ApJ, 641, 241

Rappaport, S. A., Podsiadlowski, P., \& Pfahl, E. 2005, MNRAS, 356, 401

Remillard, R. A., Muno, M. P., Morgan, E. H., \& McClintock, J. E. 2003, in ASP Conf. Ser., 308, ed. E. P. van den Heuvel, L. Kaper, E. Rol, \& R. A. M. J. Wijers, 157
Rizzi, L., Tully, R. B., Makarov, D., et al. 2007, ApJ, 661, 815

Roberts, T. P. 2007, Ap\&SS, 311, 203

Rosado, M., Ghosh, K. K., \& Fuentes-Carrera, I. 2008 [arXiv: 0803. 3003]

Ryder, S. D., Staveley-Smith, L., Malin, D., \& Walsh, W. 1995, AJ, 109, 1592

Salasnich, B., Girardi, L., Weiss, A., \& Chiosi, C. 2000, A\&A, 361, 1023

Sandage, A., \& Brucato, R. 1979, AJ, 84, 472

Schlegel, D. J., Finkbeiner, D. P., \& Davis, M. 1998, ApJ, 500, 525

Shakura, N. I., \& Syunyaev, R. A. 1973, A\&A, 24, 337

Shaposhnikov, N., \& Titarchuk, L. 2007, ApJ, 663, 445

Sirianni, M., Jee, M. J., Benítez, N., et al. 2005, PASP, 117, 1049

Soria, R. 2006 [arXiv: astro-ph/0611152]

Soria, R. 2007, Ap\&SS, 311, 213

Soria, R., \& Motch, C. 2004, A\&A, 422, 915

Soria, R., Cropper, M., Pakull, M., Mushotzky, R., \& Wu, K. 2005, MNRAS, 356,12

Soria, R., Fender, R. P., Hannikainen, D. C., Read, A. M., \& Stevens, I. R. 2006, MNRAS, 368, 1527

Soria, R., Baldi, A., Risaliti, G., et al. 2007, MNRAS, 379, 1313

Strohmayer, T. E., \& Mushotzky, R. F. 2003, ApJ, 586, L61

Strohmayer, T. E., Mushotzky, R. F., Winter, L., et al. 2007, ApJ, 660, 580

Swartz, D. A., Ghosh, K. K., Tennant, A. F., \& Wu, K. 2004, ApJS, 154, 519

Swartz, D. A., Soria, R., \& Tennant, F. T. 2008, ApJ, accepted

Tully, R. B. 1988, Nearby galaxies catalog (Cambridge and New York: Cambridge University Press), 221

van Paradijs, J., \& McClintock, J. E. 1994, A\&A, 290, 133

Walsh, J. R., \& Roy, J.-R. 1997, MNRAS, 288, 726

Zezas, A., Fabbiano, G., Rots, A. H., \& Murray, S. S. 2002, ApJ, 577, 710 